\title{
Methylmalonic aciduria articles
}

\author{
Brian Fowler
}

Published online: 16 January 2008

(C) SSIEM and Springer 2008

Methylmalonic aciduria can occur to a widely varying degree, in isolation or combined with homocystinuria in a range of conditions. These include absorption and transport disorders or defects of intracellular cellular metabolism of vitamin $\mathrm{B}_{12}$, nutritional deficiency of this vitamin, or genetic defects of methylmalonyl-CoA mutase. Recent advances have elucidated the genes and mutations responsible for most of these disorders.

The biochemically and genetically defined methylmalonic acidurias constitute an important group of potentially treatable disorders with still major diagnostic and management challenges. Furthermore, less well defined milder methylmalonic aciduria has recently been shown in some cases to be associated with SUCL mutations or methylmalonyl-CoA epimerase deficiency.

It is therefore timely and opportune that the editors of this Journal have chosen to publish two series of papers on this topic.

The articles which appear in this issue include the review "Neurodegeneration and chronic renal failure in methylmalonic aciduria-A pathophysiological approach" by Marina Morath and colleagues. This proposes and examines interesting and plausible hypotheses on pathogenetic mechanisms about which little is known. The original article "Methylmalonate inhibits succinate-supported oxygen consumption by

\section{B. Fowler $(\square)$}

Metabolic Unit, University Children's Hospital,

Basel CH-4005, Switzerland

e-mail: Brian.Fowler@unibas.ch interfering with mitochondrial succinate uptake" by Sandra Mirandola and colleagues reports interesting evidence of a possible patho-physiological role of methylmalonic acid in inhibition of succinate uptake in rat brain or muscle mitochondria. The original article "Methylmalonic acidaemia: Examination of genotype and biochemical data in 32 patients belonging to mut, cblA or cblB complementation group" by Begona Merinero and colleagues reports an interesting critical comparison of in vivo and in vitro biochemical parameters with the nature of mutations in different complementation groups.

The follow-up series will include mainly review articles (planned for issue 3, 2008) and will cover the following:

- Isolated methylmalonic acidurias in European metabolic centres (Hoerster et al)

- A review of the spectrum of MRI changes in methylmalonic aciduria (Harting et al)

- The comprehensive diagnostic approach for the reliable characterization of patients with isolated methylmalonic aciduria (Fowler et al)

- Present knowledge of potential and experience of newborn screening for the methylmalonic acidurias (Lindner et al).

We hope this series of articles will provide a solid basis to underscore further developments in this field and will serve to support future much needed prospective studies and recommendations aimed at improving the long term outcome of these life-threatening disorders. 